10 Thomsen RW, Lange P, Hellquist B, et al. Validity and underrecording of diagnosis of COPD in the Danish National Patient Registry. Respir Med 2011; 105: 1063-1068.

11 Lynge E, Sandegaard JL, Rebolj M. The Danish National Patient Register. Scand J Public Health 2011; 39: 30-33.

12 Health and Social Care Information Centre. Hospital Episode Statistics. 2013. www.hesonline.nhs.uk Date last accessed: November 8, 2013.

\title{
HIV and multidrug-resistant tuberculosis: overlapping epidemics
}

\author{
To the Editor:
}

People infected with Mycobacterium tuberculosis and HIV are much more likely to develop active tuberculosis (TB) than people with M. tuberculosis but without HIV [1]. Patients infected with multidrugresistant (MDR)-TB (defined as resistance to at least rifampicin and isoniazid, the two most powerful antiTB drugs) require longer, more expensive treatment regimens than drug-susceptible $\mathrm{TB}$, with poorer treatment success $[2,3]$. Therefore, MDR-TB poses a major challenge to the control of TB, with an estimated global disease incidence in 2012 of 450000 cases (95\% CI $300000-600000$ ) [4]. Although HIV is a powerful risk factor for all forms of TB and institutional outbreaks of MDR-TB among people living with HIV have been reported [5], population-level data on the association between HIV infection and MDR-TB are limited.

We explored the relationship between HIV infection and MDR-TB disease using data reported by member states to the World Health Organization (WHO) within the context of the Global Project on Anti-TB Drug Resistance Surveillance. The data were aggregated numbers of cases reported from either drug resistance surveys or continuous surveillance systems. Such surveys are epidemiological studies designed to measure drug resistance among a representative sample of notified pulmonary TB patients. Continuous surveillance is based on routine drug susceptibility testing of all bacteriologically confirmed TB patients. Subnational level data that were not representative of the entire country were excluded from the analysis, except for the Russian Federation and Ukraine, which are high MDR-TB burden countries for which high quality national level data were not available. The data included in the analysis met the criteria for data quality and national representativeness provided in detail elsewhere $[4,6]$. The laboratory methods used for diagnosis were endorsed by WHO.

For each country, the relationship between HIV infection and MDR-TB disease was investigated by logistic regression to calculate odds ratios and 95\% confidence intervals, using Stata (version 12; StataCorp, College Station, TX, USA). For countries with data from multiple years, robust standard errors accounted for within-country time dependencies. In order to minimise bias, data for a given year were excluded if $\leqslant 25 \%$ of reported TB patients had a documented HIV test result. 41 countries, accounting for $25 \%$ of the estimated global MDR-TB burden in 2012, met the inclusion criteria for $\geqslant 1$ year. Most were high-income countries and/or in the European Region. Only four countries were in the African region, which accounted for $75 \%$ of the global number of HIV-positive TB cases in 2012 [4]. Of these 41 countries, odds ratios could be calculated for 24 countries; the other 17 countries reported no HIV-positive MDR-TB cases (table 1). Complete HIV and MDR-TB data were available for 104781 TB patients from 1997-2012.

For 11 of the 24 countries for which the analysis was performed, HIV-positive TB patients had a significantly higher odds $(\mathrm{p}<0.05)$ of MDR-TB disease than HIV-negative TB patients (table 1$)$. Seven of these countries were in eastern Europe and central Asia: Estonia, Kazakhstan, Latvia, the Republic of Moldova, the Russian Federation, the Ukraine and Uzbekistan. For almost all of these 11 countries, the prevalence of MDR-TB among newly diagnosed TB cases (table 1) was higher than the estimated global average of $3.6 \%$ (95\% CI 2.1-5.1\%) in 2012 [4]. Although the odds ratio was highest in Kuwait, only low numbers of HIV-positive cases were reported. HIV-positive TB patients in the USA had a lower odds of MDR-TB disease than HIV-negative patients.

As data were included if $\geqslant 75 \%$ of reported TB patients had a documented HIV test, the possibility of bias due to missing data cannot be ruled out. Although a positive association between HIV infection and MDR$\mathrm{TB}$ disease was demonstrated in less than half of the countries, a recent systematic review and meta-analysis found an odds of MDR-TB in HIV-positive patients that was 1.24 times (95\% CI 1.04-1.43) higher than in 


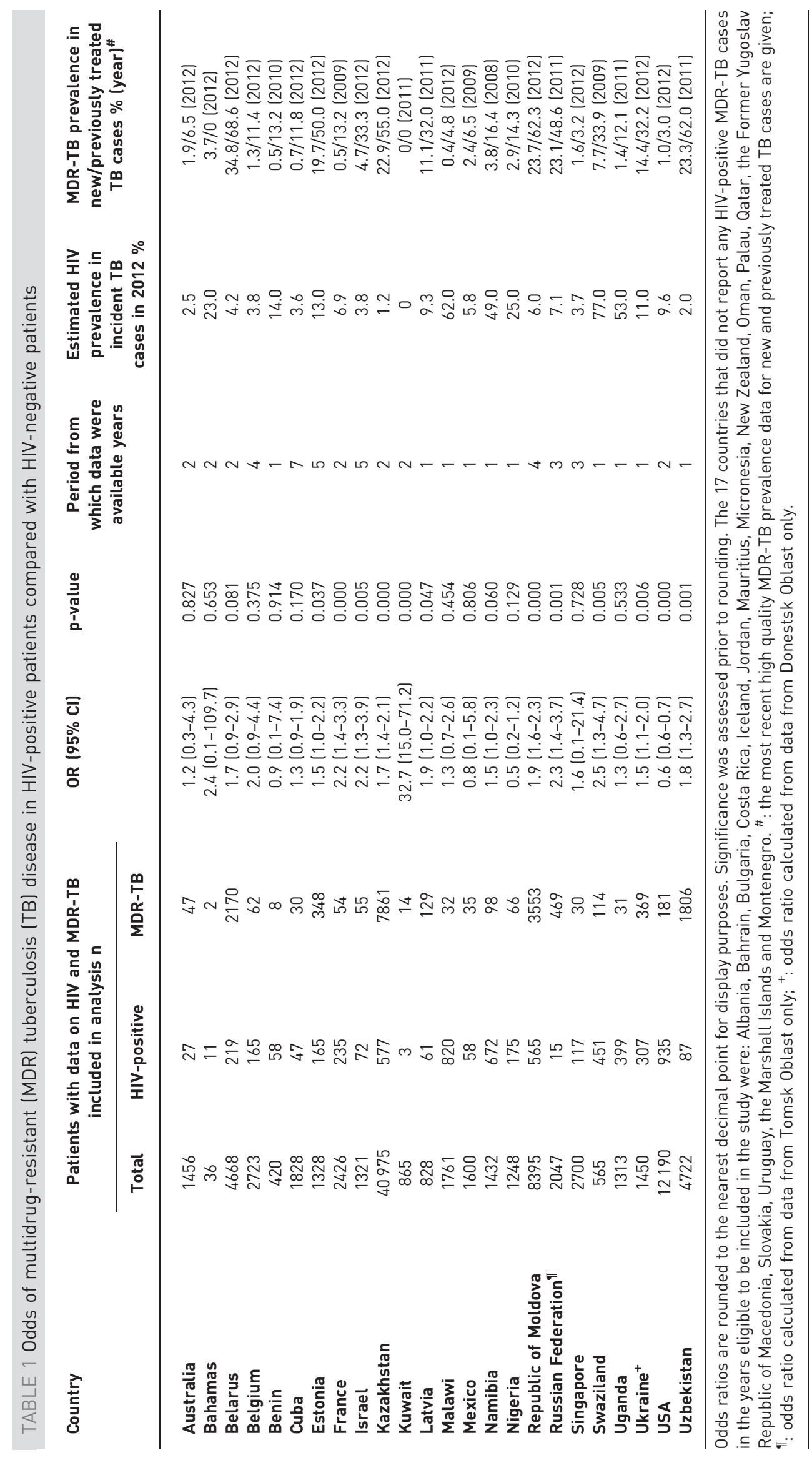


HIV-negative patients [7]. A subgroup analysis showed that this association was stronger for primary MDRTB disease than acquired MDR-TB disease, which supports previous findings [8]. However, most of these studies were from institutional settings, where outbreaks are known to occur more commonly in HIVpositive patients [9]. At the population level, a detailed analysis of surveillance data from the Republic of Moldova showed that, even after adjustment for potential confounders, a positive association HIV and MDR-TB existed [10].

Among those 11 countries with a positive association between HIV infection and MDR-TB disease, there was wide variation in the estimated prevalence of HIV among incident TB cases in 2012 (table 1) [4]. Given that the analysis was performed using aggregated rather than individual patient data, there are likely to be risk factors common to HIV-positive and MDR-TB patients that could not be explored in our analysis, such as shared behaviours or population characteristics. Further investigation is needed for specific high risk groups, such as prisoners or people living in congregate settings, miners, or injecting drug users. Additionally, the relationship between HIV and MDR-TB probably depends on the epidemiological setting. In the USA, where a negative association was observed between HIV infection and MDR-TB disease, investigation into the demographics of these patients is required in order to identify potential confounders. However, a cross-sectional study in eight states did not demonstrate a significant association between HIV infection and MDR-TB disease [11]. HIV has been shown to be an independent risk factor for drug resistance acquired during treatment in California [12].

Data were only available for three countries listed among both the 27 high MDR-TB burden countries and 41 high TB/HIV burden countries: Nigeria, the Russian Federation and the Ukraine. In order to better understand the relationship between HIV infection and MDR-TB, more high quality data are needed from other high burden countries. This includes the Democratic Republic of Congo, Ethiopia, Myanmar, South Africa and Vietnam. In these five countries, the percentage of estimated cases of MDR-TB that were detected and notified in 2012 ranged from just 2.2\% to 30\% and the percentage of TB patients with a known HIV status in 2012 ranged from 13 to 66\%, excluding South Africa [4]. This is probably a reflection of low levels of testing for MDR-TB and HIV among TB patients as well as underreporting. Priorities in these countries should include integrated TB and HIV services and programmes, with universal access to HIV testing and routine and prompt TB drug susceptibility testing for patients suspected to have MDR-TB. This must be underpinned by greater awareness of healthcare workers, increased laboratory capacity, and improved data management and reporting [13]. The roll-out of rapid diagnostic tests and the use of MDRTB treatment models that include community-based care could rapidly increase MDR-TB case notification and enrolment on second-line treatment, ultimately limiting spread [14]. Development of region- and country-specific comprehensive responses, including research for better diagnosis, treatment and scale-up of quality services, is essential [15].

○

@ERSpublications

Development of region- and country-specific comprehensive responses to HIV/MDR-TB co-infection is essential http://ow.ly/tgkvt

Anna S. Dean, Matteo Zignol, Dennis Falzon, Haileyesus Getahun and Katherine Floyd

Global Tuberculosis Programme, World Health Organization, Geneva, Switzerland.

Correspondence: Katherine Floyd, World Health Organization, 20 Avenue Appia, 1211 Geneva, Switzerland.

E-mail: floydk@who.int

Received: Dec 132013 | Accepted after revision: Jan 252014 | First published online: February 132014

Conflict of interest: None declared.

Acknowledgements: All authors are staff members of WHO. The authors alone are responsible for the views expressed in this publication and they do not necessarily represent the decisions or policies of WHO.

This article is one of a selection of articles published as ERJ Open papers, as part of an initiative agreed between the European Respiratory Society and the World Health Organization.

\section{References}

1 Selwyn PA, Hartel D, Lewis VA, et al. A prospective study of the risk of tuberculosis among intravenous drug users with human immunodeficiency virus infection. N Engl J Med 1989; 320: 545-550.

2 Migliori GB, Sotgiu G, Gandhi NR, et al. Drug resistance beyond extensively drug-resistant tuberculosis: individual patient data meta-analysis. Eur Respir J 2013; 42: 169-179.

3 Falzon D, Gandhi N, Migliori GB, et al. Resistance to fluoroquinolones and second-line injectable drugs: impact on multidrug-resistant TB outcomes. Eur Respir J 2013; 42: 156-168.

4 World Health Organization. Global Tuberculosis Report 2013. www.who.int/tb/publications/global_report/en/ index.html Date last accessed: November 1, 2013. Date last updated: 2013. 
5 Frieden TR, Sherman LF, Maw KL, et al. A multi-institutional outbreak of highly drug-resistant tuberculosis: epidemiology and clinical outcomes. JAMA 1996; 276: 1229-1235.

6 World Health Organization. Guidelines for surveillance of drug resistance in tuberculosis. 4th Edn. http://whqlibdoc. who.int/publications/2009/9789241598675_eng.pdf Date last accessed: August 6, 2013. Date last updated: 2009.

Mesfin YM, Hailemariam D, Biadglign S, et al. Association between HIV/AIDS and multi-drug resistance tuberculosis: a systematic review and meta-analysis. PLoS ONE 2014; 9: e82235.

8 Suchindran S, Brouwer ES, Van Rie A. Is HIV infection a risk factor for multi-drug resistant tuberculosis? A systematic review. PLoS ONE 2009; 4: e5561.

9 Wells CD, Cegielski JP, Nelson LJ, et al. HIV infection and multidrug-resistant tuberculosis: the perfect storm. J Infect Dis 2007; 196: Suppl. 1, S86-S107.

10 Jenkins HE, Plesca V, Ciobanu A, et al. Assessing spatial heterogeneity of multidrug-resistant tuberculosis in a highburden country. Eur Respir J 2012; 42: 1291-1301.

11 Moonan PK, Teeter LD, Salcedo K, et al. Transmission of multidrug-resistant tuberculosis in the USA: a crosssectional study. Lancet Infect Dis 2013; 13: 777-784.

12 Porco TC, Oh P, Flood JM. Antituberculosis drug resistance acquired during treatment: an analysis of cases reported in California, 1994-2006. Clin Infect Dis 2013; 56: 761-769.

13 Falzon D, Jaramillo E, Wares F, et al. Universal access to care for multidrug-resistant tuberculosis: an analysis of surveillance data. Lancet Infect Dis 2013; 13: 690-697.

14 Nardell E, Dharmadhikari A. Turning off the spigot: reducing drug-resistant tuberculosis transmission in resourcelimited settings. Int J Tuberc Lung Dis 2010; 14: 1233-1243.

15 Getahun H, Gunneberg C, Granich R, et al. HIV infection-associated tuberculosis: the epidemiology and the response. Clin Infect Dis 2010; 50: S201-S207.

\section{Rapid diagnosis of tuberculosis using ex vivo host biomarkers in sputum}

\section{To the Editor:}

Tuberculosis continues to be a major public health problem in developing countries [1]. One of the roadblocks in reducing tuberculosis transmission is the lack of accurate laboratory-free diagnostic tests for use at the point of care. If tuberculosis is to be eliminated, we need a robust, low-cost and safe point-of-care diagnostic test, which in turn requires identification of appropriate biomarkers [2]. Rapid tests based on microfluidics (lateral flow tests) hold great promise for tuberculosis diagnostics. They are easy to use, cheap, provide an answer within minutes, do not require specialised equipment and are stable at room temperature, making them ideal for use in high tuberculosis burden, resource-poor settings. To date, however, no such test has been developed for tuberculosis due to lack of sensitivity related to the markers and/or sample type. Development of tests based on host biomarkers requires evaluation of different sample types [3-5] and markers other than interferon (IFN)- $\gamma$ [5] to provide differential diagnosis of active tuberculosis, latent infection and other respiratory disorders. We have previously shown that a combination of three host factors in pleural fluid resulted in $96 \%$ correct classification of tuberculosis among other respiratory diseases (ORD) (including bacterial pneumonia) regardless of HIV status [6]. However, this sample type is not easy to obtain and we therefore wanted to determine if we could use ex vivo sputum, which is noninvasive and easy to obtain in adult pulmonary tuberculosis patients.

Subjects were consecutively recruited from the outpatient clinic and ward at the Medical Research Council Unit, Fajara, the Gambia. All subjects were adults ( $\geqslant 18$ years of age) with symptoms suggestive of tuberculosis. Subjects were subsequently classified into two groups: those with culture-confirmed tuberculosis and those with ORD. 75\% of the tuberculosis and 50\% of the ORD group were positive by the IFN- $\gamma$ QuantiFERON test (Qiagen, Hilden, Germany). Samples were collected concomitantly from the same patient. Serum was collected using serum Vacutainers (BD, Franklin Lakes, NJ, USA) following centrifugation and saliva was collected using a passive drool technique. $1 \mathrm{~mL}$ of fresh sputum was digested for $15 \mathrm{~min}$ at room temperature with $0.1 \%$ dithiothreitol. An equal volume of PBS was added, the samples were centrifuged $(600 \times g$ for $5 \mathrm{~min})$, and the supernatants were collected and stored at $-20^{\circ} \mathrm{C}$. Undiluted heparinised blood ( $450 \mu \mathrm{L}$ per well) was stimulated with purified protein derivative (PPD) (Statens Serum Institut, Copenhagen, Denmark) or ESAT-6 (6-kDa early secreted antigen)/CFP-10 (10-kDa culture filtrate protein) at a final concentration of $10 \mu \mathrm{g} \cdot \mathrm{mL}^{-1}$. After $24 \mathrm{~h}$ incubation (at $37^{\circ} \mathrm{C}$ and $5 \%$ carbon dioxide), supernatants were harvested and analysed by multiplex cytokine array. Samples were analysed using either a custom 13-plex (stimulated blood) or 27-plex Bio-Plex (serum, saliva and sputum) pre-mixed cytokine/ 